DOI: https://doi.org/10.46296/yc.v5i8edespen.0091

\title{
RESILIENCIA EN LOS MIEMBROS DEL CUERPO DE BOMBEROS DURANTE EL CONFINAMIENTO POR COVID-19 EN LA PARROQUIA ABDÓN CALDERÓN
}

\section{RESILIENCE IN THE MEMBERS OF THE FIRE DEPARTMENT DURING THE CONFINEMENT BY COVID-19 IN THE ABDÓN CALDERÓN PARISH}

\author{
Intriago-Intriago Gabriela Stefanía ${ }^{1 *}$; Saldarriaga-Villamil Kasandra Vanessa ${ }^{2}$ \\ 1,2 Universidad Técnica de Manabí, UTM. Portoviejo, Ecuador. \\ *Correo: gabrin2008@hotmail.com
}

\begin{abstract}
Resumen
La resiliencia es un término que se refiere a la capacidad de una persona o grupo de resistir y recuperarse de impactos o presión. El objetivo de la investigación es analizar los niveles de resiliencia de los miembros del cuerpo de bomberos de la parroquia Abdón Calderón durante el confinamiento por Covid-19. El enfoque investigativo es mixto, de estructura cualitativa y cuantitativa, se describe el estado situacional, mediante las técnicas inductivas y deductivas, se utiliza como instrumento la escala de Resiliencia SV-RES. Los resultados indican que los miembros del cuerpo de bomberos de la compañía Abdón Caderón han tenido normas muy estrictas de bioseguridad como la encontrarse en total confinamiento en el periodo declarado como emergencia sanitaria, sin embargo, manejan niveles de resiliencia altos, lo cual lo asocian a las capacitaciones recibidas, el entrenamiento laboral que tienen para atender situaciones de emergencia, peligro y rescate, así mismo al apoyo de sus familias con quienes han tenido que convivir de una forma hasta cierto punto aislada para salvaguardar su salud y la de los suyos.
\end{abstract}

Palabras clave: Nivel de resiliencia; confinamiento; bioseguridad; cuerpo de bombero.

\begin{abstract}
The resilience is a term that refers to the ability of a person or group to resist and recover from impacts or pressure. The objective of the research is to analyze the levels of resilience of the members of the Abdon Calderon parish fire department during the confinement by Covid-19. The investigative approach is mixed, of qualitative and quantitative structure, the situational state is described, by means of the inductive and deductive techniques, the SV-RES Resilience Scale is used as an instrument. The results indicate that the members of the fire department of the company Abdon Caderon have had very strict norms of biosecurity, such as being in total confinement during the period declared as a sanitary emergency. However, they manage high levels of resilience, which they associate with the training received, the labor training they have to attend to emergency, danger and rescue situations, as well as the support of their families with whom they have had to live in a somewhat isolated way to save their health and that of their loved ones.
\end{abstract}

Keywords: Level of resilience; confinement; biosafety; fire department

Información del manuscrito:

Fecha de recepción: 16 de noviembre de 2020.

Fecha de aceptación: 22 de enero de 2021.

Fecha de publicación: 29 de enero de 2021. 


\section{Introducción}

La situación de pandemia y el confinamiento por el COVID-19 es, sin duda alguna es el evento más extremo al que ha tenido que enfrentarse la sociedad en lo que va del siglo. Tanto por su extensión, que ha afectado a muchas personas en todo el mundo, como por su duración de meses, su previsible continuidad y sus consecuencias. El escenario de excepcionalidad derivado de la pandemia ha implicado todas las áreas del funcionamiento de la sociedad como son el sanitario, económico, familiar y social. Los decretos de los estados han ordenado el confinamiento a las personas, y han dado prioridad a las ocupaciones y actividades de forma virtual, sin embargo, no en todos los ámbitos y trabajos se puede utilizar la virtualidad, como en el caso del personal de salud, y en los grupos de emergencia y de rescate como es el cuerpo de bomberos, quienes debieron mantener sus actividades de forma normal dentro de las compañías, aunque le añadieron tareas laborales debido al covid-19, sin embargo debieron mantenerse en confinamiento en sus casas después de salir de sus turnos de trabajo, el presente trabajo pretende analizar los niveles de resiliencia de los miembros del cuerpo de bomberos de la Parroquia Abdón Calderón, durante el confinamiento, en el periodo marzo-agosto de 2020.

El confinamiento es una intervención que se aplica a nivel comunitario cuando las medidas de bioseguridad han sido insuficientes para contener el contagio de una enfermedad (M. Cetron, J. Landwirth, 2005). Consiste en un estado donde se combinan estrategias para reducir las interacciones sociales como el distanciamiento social, el uso obligatorio de mascarillas, restricción de horarios de circulación, suspensión del transporte, cierre de fronteras, etcétera (A. Wilder-Smith, D.O. Freedman, 2019). Los conceptos como aislamiento social y confinamiento denotan medidas de contención para evitar la propagación de una enfermedad contagiosa. La cuarentena se aplica a una persona o un grupo ante sospecha de que uno de ellos está infectado, y el aislamiento se da cuando se ha confirmado el contagio. Por su parte, el distanciamiento social implica 
separarse y conservar un espacio físico determinado entre las personas, y el confinamiento es un plan de intervención comunitario que implica estar refugiado el tiempo que más se pueda, acatando nuevas formas de restricción social.

Por otro lado, para Wolfgang Wagner y Rosanne Anholt (2016), definen la "Resiliencia" en el sentido literal como un término referente a la capacidad de un cuerpo o materia de volver a su estado original después de estar sometido a un impacto o presión. En base a esto, en cuanto a las personas como tales, se podría definir entonces la resiliencia como la capacidad de una persona o grupo de resistir y recuperarse de impactos.

El hecho de que la resiliencia considere un aspecto dinámico y afectado por diversas situaciones traslada el debate, según SandínEsteban \& Sánchez-Martí (2015) en torno a interrogantes como si la resiliencia es un proceso 0 un producto, si los que tienen más peso en los individuos son los factores externos o los internos, etc. Gran parte de las investigaciones enfatizan la importancia de la resiliencia para el equilibrio emocional por su aporte en la superación de situaciones difíciles de tipo emocional, mental, educativo, económico (Cachón et al., 2014; Sandín-Esteban \& Sánchez-Martí, 2015; Sandoval \& Cortés, 2012; Tomás-Miquel et al., 2016).

La resiliencia se relaciona también con las actitudes optimistas, la satisfacción con la propia vida y el bienestar percibido (Cazalla-Luna \& Molero, 2016; Denovan \& Macaskil, 2017). Para González-Muzzio (2013), tras un evento traumático aparecen conductas emergentes que movilizan los recursos de los grupos para hacer frente de la mejor manera a la nueva situación. Los medios digitales facilitan la movilización amplia y diversa del capital social de cada sujeto y comunidad, para actuar de un modo resiliente mejorando su respuesta (Brewer et al., 2019).

A causa de la situación actual de pandemia generada por el COVID19, se han desarrollado varios estudios sobre resiliencia como el llevado a cabo en el ámbito sanitario (Bryce et al., 2020), otro estudio es el del impacto psicológico y social en la 
vida cotidiana (Arrosi et al., 2020), la investigación realizada por Cleland et al., 2020, sobre el modo en el que la comunidad universitaria desarrolla diferentes estrategias para ajustarse a las nuevas exigencias de la educación virtual, entre otros estudios que pretender analizar la resiliencia en cada uno de los aspectos de la vida de la sociedad.

La resiliencia es una construcción que necesita preparación, el objetivo es preparar a los actores para una crisis mediante la reducción del efecto sorpresa, y se debe cuidar que este trabajo de preparación no sea inquietante. La resiliencia de los servicios de rescate es esencial, sin embargo, es necesario considerar que la resiliencia no solo consiste en asegurar el buen funcionamiento de los colaboradores dentro de la estación bomberil, sino que debe ser un proceso inclusivo que abarque su bienestar integral y que esto le permita desarrollar sus actividades en el ámbito laboral, familiar y social.

\section{Objetivo}

El objetivo de la investigación fue analizar los niveles de resiliencia desarrollado por los miembros del Cuerpo de Bomberos de la Parroquia
Abdón Calderón, para lograr cumplirlo se identificaron las medidas de prevención y de bioseguridad utilizadas por ellos, sus horarios de trabajo, y luego se procedió a identificar el nivel de resiliencia durante el confinamiento por COVID-19.

\section{Metodología}

La investigación se planteó desde el enfoque mixto, cual-cuantitativo. Se procedió a través del soporte descriptivo y los métodos de inducción y deducción. Para su desarrollo se empezó identificando los horarios de trabajo del personal, cuántos grupos eran y cómo rotaban durante este periodo, cuál fue su carga laboral durante este tiempo, qué medidas de bioseguridad y de prevención adoptaron, qué medidas administrativas se aplicaban para quienes las incumplían. La técnica utilizada fue la de la encuesta, y como instrumento se aplicó el test de Resiliencia SV-RES, el cual cumplía con el objetivo de medir los niveles de resiliencia que tienen los miembros de la estación de bomberos.

Población: En la correlación e interpretación de los datos se 
reconocieron a 10 miembros de la Compañía del Cuerpo de Bomberos de la parroquia Abdón Calderón, del cantón Portoviejo, de la provincia de Manabí. Como muestra se tomaron las 10 personas que laboran en la estación.

\section{Análisis e interpretación de resultados}

\subsection{Análisis situacional descriptivo:}

La compañía del cuerpo de Bomberos Portoviejo es una institución al servicio de la ciudadanía, fue creada el 16 de junio de 1888, desde entonces ha prestado sus servicios en la atención de emergencias, ya sean incendios, rescates, salvamentos, emergencias médicas prehospitalarias, incidentes con materiales peligrosos y como no mencionar su razón de ser, la prevención de accidentes y siniestros como los antes detallados. Ante los datos expuestos, se logró reconocer que en el cantón Portoviejo existen 9 estaciones del Cuerpo de Bomberos, una de ellas es la de la parroquia Abdón Calderón, la cual está compuesta por 10 colaboradores, 8 hombres y dos mujeres, quienes prestan sus servicios de lunes a domingo las 24 horas del día, los turnos de trabajo, que son reconocidos como guardias, están clasificados en 3 grupos, 2 de 3 personas y 1 grupo de 4 , cada guardia es de 24 horas dentro de la institución y descansan 48 horas.

En el periodo declarado como emergencia sanitaria, sus horarios de trabajo se mantuvieron de igual manera, les fueron designadas actividades como repartir agua a los sectores donde no llegaba, desinfectar las calles, y atender emergencias de salud. Desde el comienzo de este periodo y aún en la actualidad mantienen estrictas normas y protocolos de bioseguridad, como el uso de mascarillas, alcohol, toma de la temperatura, atención médica frecuente, se les realiza pruebas del covid-19, y se les prohíbe la salida a reuniones, festejos, aglomeraciones y la salida del cantón de residencia.

\subsection{Test de Resiliencia SV-RES}

Para realizar la aplicación del test, se procedió a determinar la muestra de estudio que constó de 10 personas en donde se correlaciona la siguiente tabla sobre la escala percentil que se describe a continuación. 
Tabla 1. Cuadro de interpretación de la escala percentil.

\begin{tabular}{|c|c|c|}
\hline \multicolumn{3}{|c|}{ INTERPRETACIÓN DEL PUNTAJE PERCENTIL } \\
\hline Puntaje percentil & $0-25$ & Bajo \\
\hline Puntaje percentil & $26-75$ & Promedio \\
\hline Puntaje percentil & $76-100$ & Alto \\
\hline
\end{tabular}

Fuente: Test SV-RE S.

Tabla 2. Análisis de los resultados de la prueba SV-RES.

\begin{tabular}{|c|l|c|c|}
\hline PUNTAJE DIRECTO & PUNTAJE EN VALOR PERCENTIL & FRECUENCIA & PORCENTAJE \\
\hline $0-25$ & PUNTAJE PERCENTIL 0-25 = Bajo & 0 & Bajo \\
\hline $26-75$ & PUNTAJE PERCENTIL 26-74 = Promedio & 1 & Promedio \\
\hline $76-100$ & PUNTAJE PERCENTIL 75-99 = Alto & 9 & Alto \\
\hline & TOTAL & 10 & \\
\cline { 2 - 4 }
\end{tabular}

Fuente: Encuesta aplicada al personal del cuerpo de bomberos, Abdón Calderón. Elaboración: Las investigadoras (2020).

Figura 1. Nivel de resiliencia en los miembros del cuerpo de Bomberos.

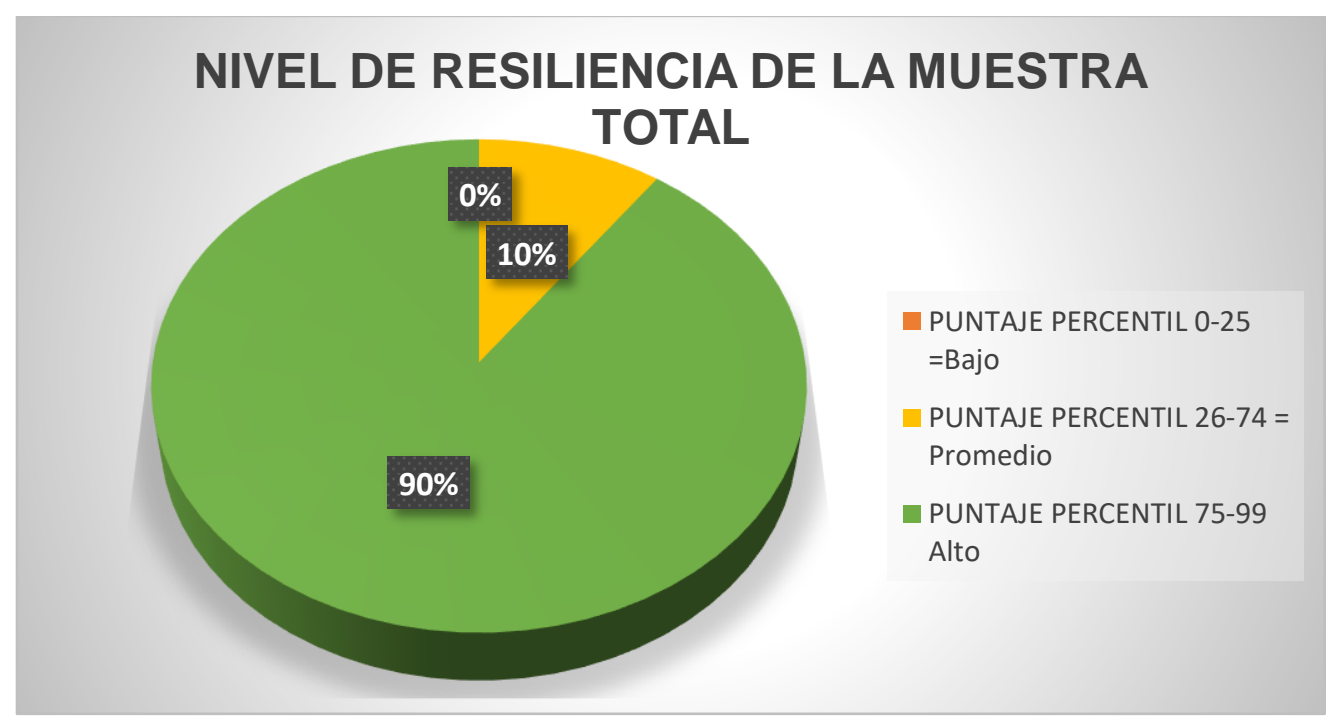

Fuente: Encuesta aplicada al personal del cuerpo de bomberos, Abdón Calderón. Elaboración: Las investigadoras (2020).

En la tabla número 2 , se puede observar que los miembros del cuerpo de bomberos que participaron en el estudio, según el test SV-RES, tienen un nivel de resiliencia alto, esto se debe a las capacitaciones recibidas y a la formación que tienen en su proceso de aceptación e ingreso a la compañía del cuerpo de bomberos, 
pues ellos son parte de los grupos de rescate y atención a emergencias, por lo que tienen que estar preparados para situaciones adversas, sin embargo en tiempos de pandemia algunas cosas han cambiado y la sociedad no estaba preparado para vivir algo así como el confinamiento, pero a pesar de esto, ellos han contado con las medidas de bioseguridad necesarias, capacitaciones en cuanto al tema y el apoyo de sus familias para cumplir con las medidas establecidas, entre ellas el confinamiento.

Tabla 3. Nivel de Resiliencia "Yo soy-yo estoy".

\begin{tabular}{|c|c|}
\hline FACTOR & FRECUENCIA \\
\hline F1 IDENTIDAD & 10 \\
\hline F2 AUTONOMÍA & 10 \\
\hline F3 SASTISFACCIÓN & 9 \\
\hline F4 PRAGMATISMO & 9 \\
\hline
\end{tabular}

Fuente: Encuesta aplicada al personal del cuerpo de bomberos, Abdón Calderón.

Elaboración: Las investigadoras (2020).

Figura 2. "Yo soy- yo estoy".

\section{YO SOY- YO ESTOY}

\section{$24 \%$}

$24 \%$

\section{$26 \%$}

$26 \%$
- F1 IDENTIDAD

- F2 AUTONOMÍA

F3 SASTISFACCIÓN

F4 PRAGMATISMO

Fuente: Encuesta aplicada al personal del cuerpo de bomberos, Abdón Calderón.

Elaboración: Las investigadoras (2020).

El primer nivel de resiliencia se estable en la tabla número 3 , la cual establece que los miembros del cuerpo de bomberos tienen una buena autodefinición y caracterización personal, lo cual representa su identidad. Manifiestan tener autonomía al ser independientes al actuar y mantener el control interno para saberlo hacer. También muestran un nivel de satisfacción elevado, una buena 
percepción de los logros que han tenido y una adaptación afectiva a sus condiciones ambientales. El pragmatismo se reconoció como el sentido práctico que tienen para evaluar y enfrentar los problemas, así como su orientación hacia la acción.

Tabla 4. Nivel de Resiliencia "Yo tengo".

\begin{tabular}{|c|c|}
\hline FACTOR & FRECUENCIA \\
\hline F5 VINCULOS & 10 \\
\hline F6 REDES & 9 \\
\hline F7 MODELOS & 8 \\
\hline F8 METAS & 9 \\
\hline
\end{tabular}

Fuente: Encuesta aplicada al personal del cuerpo de bomberos, Abdón Calderón.

Elaboración: Las investigadoras (2020).

Figura 3. "Yo tengo".

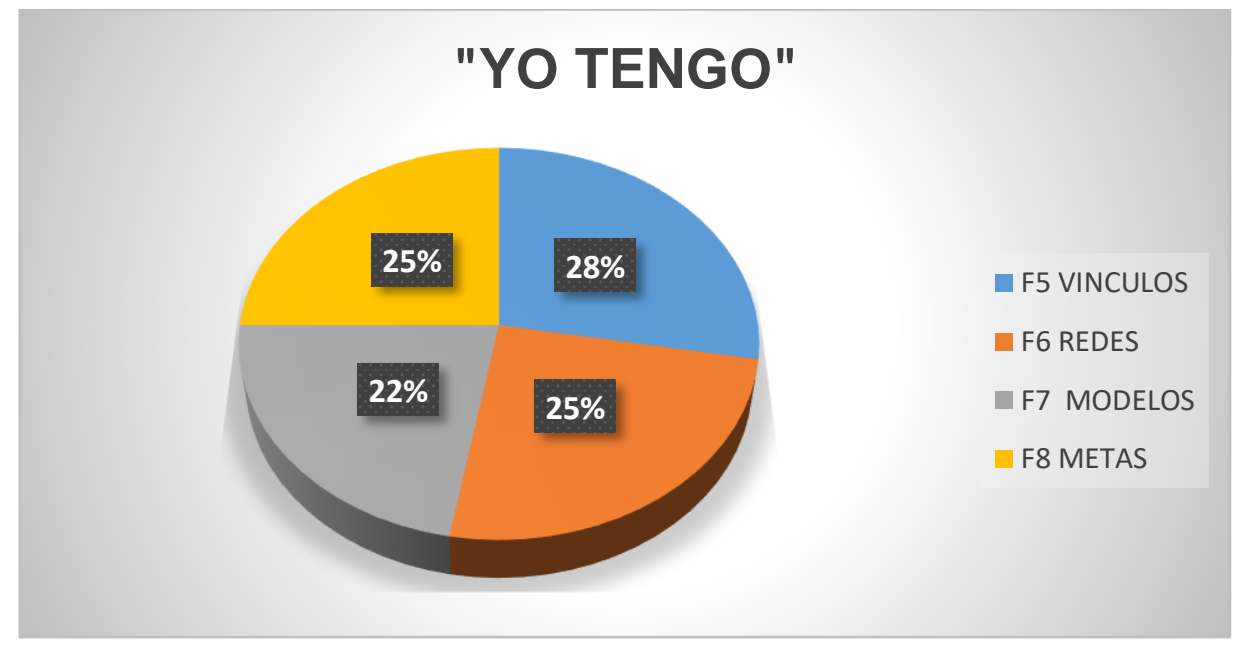

Fuente: Encuesta aplicada al personal del cuerpo de bomberos, Abdón Calderón Elaboración: Las investigadoras (2020).

En la tabla 4 se muestra el nivel de resiliencia en cuanto a sus vínculos, los cuales son las condiciones estructurales que le han servido de base para la formación de su personalidad. Manifiestan tener el apoyo de su familia para superar las adversidades. En cuanto a los modelos para la superación de sus problemas consideran que son sus experiencias, que los guían y hacen aprender para corregir, puesto que sus metas están establecidas proyectando su bienestar hacia el futuro. 
Tabla 5. Nivel de resiliencia "Yo puedo".

\begin{tabular}{|c|c|}
\hline FACTOR & FRECUENCIA \\
\hline F9 AFECTIVIDAD & 9 \\
\hline F10 AUTOEFICACIA & 10 \\
\hline F11 APRENDIZAJE & 10 \\
\hline F11 APRENDIZAJE & 10 \\
\hline
\end{tabular}

Fuente: Encuesta aplicada al personal del cuerpo de bomberos, Abdón Calderón Elaboración: Las investigadoras (2020).

Figura 4. "Yo puedo".

\section{YO PUEDO}

$26 \% \quad 23 \%$

$26 \% \quad 25 \%$
F9 AFECTIVIDAD

- F10 AUTOEFICACIA

F11 APRENDIZAJE

F11 APRENDIZAJE

Fuente: Encuesta aplicada al personal del cuerpo de bomberos, Abdón Calderón Elaboración: Las investigadoras (2020).

En la tabla número 5 se expone el nivel de resiliencia en cuanto la afectividad, donde hacen un reconocimiento de su vida emocional y en cómo expresan sus afectos, consideran tener autoeficacia para resolver problemas responsabilizarse por sus actos sobre todo en estos tiempos de pandemia donde el autocuidado es muy importante, además del aprendizaje adquirido en este periodo que les ha generado capacidad de crear respuestas alternativas y planificar sus acciones.

\section{Conclusiones}

Los resultados de la investigación permitieron analizar los niveles de resiliencia desarrollados por los miembros del cuerpo de bombero durante el confinamiento por el Covid-19, si bien es cierto la situación de pandemia generada por el coronavirus (Covid-19), tomó por 
sorpresa a la sociedad en general, pues ningún estado estaba preparado para la inestabilidad sanitaria provocada por el virus que ha afectado y sigue afectando a todos los países del mundo generando una crisis sanitaria, económica y hasta social, al tener que tomar el aislamiento $y$ el confinamiento como una de las medidas primordiales de prevención.

En base a esto, se han enfrentado situaciones negativas como el confinamiento antes expuesto, los miembros del cuerpo de bomberos de la compañía de Abdón Calderón manejan niveles de resiliencia altos, esto se le atribuye a que debido a su práctica profesional han tenido que capacitarse y formarse para atender emergencias y situaciones negativas teniendo que enfrentarlas estratégicamente, además del apoyo recibido por sus familiares $y$ comunidad ya que su labor siempre es estar prestos a colaborar con la sociedad, siendo así su nivel de vinculación con la sociedad un apoyo para desarrollar su trabajo y superar las dificultades que se les presenten en su vida tanto laboral como personal.

\section{Bibliografía}

Arrossi, S., Ramos, S., Paolino, M., Binder, F., Perelman, L. \& Kruptizki, H. (2020). Estudio TIARA. Primer avance de resultados. Centro de 98 Aznar Sala - COVID-19 y Educación Secundaria en España Estudios de Estado y Sociedad (CEDES). Recuperado de: http://repositorio.cedes.org/ha ndle/123456789/4534

Brewer, M.; Van Kessel, G.; Sanderson, B.; Naumann, F.; Lane, M.; Reubenson, A. \& Carter, A. (2019). Resilience in higher education students: a scoping review. Higher Education Research \& Development, 38(6), 11051120. Recuperado de: https://doi.org/10.1080/07294 360.2019 .1626810

Bryce, C., Ring, P., Ahsby, S., \& Wardman, J. K. (2020). Resilience in the face of uncertainty: early lessons from the COVID-19 pandemic. Journal of Risk Research. Recuperado de: https://doi.org/10.1080/13669 877.2020.1756379

Cachón, J., López, I., San Pedro, M. B., Zagalaz, M. L., \& González, C. (2020). The Importance of the Phoenix Bird Technique (Resilience) in Teacher Training: CD-RISC Scale

Validation. 
Sustainability, 12(3), 1002. Recuperado:

https://doi.org/10.3390/su120 31002

Cazalla-Luna, N., y Molero, D. (2016). Inteligencia emocional percibida, disposición al optimismo-pesimismo, satisfacción vital y personalidad RISE - Special Issue 99 de docentes en su formación inicial. Revista de Investigación Educativa, 34(1), 241-258. Recuperado de:

https://doi.org/10.6018/rie.34. 1.220701

Cetron, M. Landwirth, J. (2005). Public health and ethical considerations in planning for quarantine. Yale J Biol Med., 78 (2005), pp. 325-330

Cleland, C., McKimm, J., Fuller, R., Taylor, D. \& Janczukowicz, J. (2020). Adapting to the impact of COVID-19: Sharing stories, sharing practice. Medical Teacher. Recuperado de: https://doi.org/10.1080/01421 59X.2020.1757635

Denovan, A., y Macaskill, A. (2017). Stress and subjective wellbeing among first year UK undergraduate students. Journal of Happiness Studies, 18(2), 505-525. Recuperado de:

https://doi.org/10.1007/s1090 2-016-9736
González-Muzzio, C. (2013). El rol del lugar y el capital social en la resiliencia comunitaria posdesastre. Aproximación mediante un estudio de caso después del terremoto del 23/F. EURES, Revista Latinoamericana de Estudios Urbanos Regionales, 39(117), 25-48. Recuperado de: http://dx.doi.org/10.4067/S02 50-71612013000200002

Sandín-Esteban, M. P. \& SánchezMartí, A. (2015). Resiliencia y éxito escolar en jóvenes inmigrantes. Infancia y Aprendizaje: Journal for the Study of Education and Development. 38(1), 175-211. Recuperado de: https://doi.org/10.1080/02103 702.2015.1009232

Sandoval-Hernández, A., \& Cortes, D. (2012). Factors and conditions that promote academic resilience: A crosscountry perspective. Conferencia presentada en la Conferencia Anual de la Sociedad Internacional de Educación Comparada. Puerto Rico

Tomás-Miquel, J.V.; Nicolau-Juliá, D. \& Expósito-Langa, M. (2016). The social relations of university students: intensity, interaction and association with academic performance. Cultura y Educación, 28(4), 667-701. Recuperado de: 
https://doi.org/10.1080/11356 405.2016.1237340

Wilder-Smith, D.O. Freedman. (2020). Isolation, quarantine, social distancing and community containment: Pivotal role for old-style public health measures in the novel coronavirus (2019-nCoV) outbreak. J Travel Med., 27 (2020), pp. 1410.1093/jtm/taaa020

Wolfgang Wagner \& Rosanne Anholt (2016). La resiliencia como el nuevo leitmotiv de la estrategia global de la UE: ¿pragmático, problemático o prometedor? Política de seguridad contemporánea (Resilience as the EU Global Strategy's new leitmotif: pragmatic, problematic or promising? Contemporary Security Policy, 37:3, 414430 , DOI: 10.1080/13523260.2016.122 8034. 